\title{
Serum Level of Periostin and Cardiac Function in Patients with Psorasis \\ M.S.El Bahrawy ${ }^{1}$, S.I.Farg ${ }^{2}$, O.S.El-Shimi ${ }^{3}$, A.I.Mustafa ${ }^{3}$, A.A.El-Taweel ${ }^{4}$ \\ ${ }^{1}$ Kafr shokr Hospital, M.M.C.H, Benha Univ., Benha, Egypt \\ ${ }^{2}$ Cardiology Dept., Benha Univ., Benha, Egypt \\ ${ }^{3}$ Clinical and Chemical Pathology Dept., Benha Univ., Benha, Egypt \\ ${ }^{4}$ Dermatology, Venereoloy and Andrology Dept., Benha Univ., Benha, Egypt \\ E-Mail:Moshira.bahrawy@gmail.com
}

\begin{abstract}
Psoriasis is a common chronic immune mediated relapsing and remitting, inflammatory skin diseases causing significant impairment of quality of life, at least to the same extent as other major medical diseases. About 2\% of the population in Europe and the United States are affected by psoriasis. the present work aim to asses serum level of periostin in psoriatic patients, in an attempt to elucidate its role in the disease pathogenesis in psoriatic patient. fifty adult patients with psoriasis vulgaris (PV) as a disease control and30 healthy controls were determined their serum level of periostin using An enzyme-linked immunosorbent assay. serum levels of periostin were significantly increased in psoriatic patients, with a significant positive correlation to the disease severity when compared to the healthy controls. In conclusion, periostin may play a role in the pathogenesis of PV.
\end{abstract}

Keywords: Psoriasis vulgaris, Periostin.

\section{Introduction}

Psoriasis is one of the most prevalent chronic inflammatory immune mediated skin diseases causing significant impairment of quality of life, at least to the same extent as other major medical diseases [1]. It is characterized by periods of spontaneous remission and exacerbations that affect approximately $2-3 \%$ of the population worldwide [2]. Psoriasis is considered to be a Thelper (Th) cell mediated disease in which Th1, Th17 and Th22 cell populations are expanded and stimulated to release inflammatory cytokines, including tumour necrosis factor-a (TNFa), interleukin (IL)-17 and IL22 [3]. Periostin secreted from fibroblasts was required for IL- $1 \alpha$-induced IL-6 production and enhanced IL-6 production by activation of the NF- $\mathrm{KB}$ pathway synergistically with IL- $1 \alpha$. Thus, the combination of an autocrine loop of periostin and a paracrine loop composed of IL- $1 \alpha$ and IL- 6 regulates keratinocyte proliferation and differentiation in the epithelial-mesenchyme linteraction.Therefore induction of IL-6 expression in fibroblasts is one of the mechanisms by which periostin promotes keratinocyte proliferation [4].

\section{Patients and methods}

Case- control study. This study was carried out on 50 psoriasis patients (Group A) and 30 age and sex matched apparently healthy individuals as controls (Group B). All patients recurrited from the outpatient clinic of Dermatology and Andrology Department of Banha University Hospitals in the period between January 2018 to January 2019. All patients were subjected to full history taking including name, age, sex, occupation, residence, special habits of medical importance, marital and socioeconomic status, onset, course and duration of psoriasis, history of medications (type and duration), associated systemic diseases (DM, dyslipidemia, HTN or
CVD), endocrinal problems and previous surgery and history of previous treatment of PS (type, dose and duration) or Family history of PS, Clinical Examination to assess psoriasis severity by Psoriasis Area Severity Index (PASI) scoring [5]. PASI, a score used for determination of the severity of psoriasis in relation to three parameters including erythema (E), infiltration (I) and desquamation (D). All studied subjects were tested for serum level of Periostin by A doubleantibody sandwich ELISA (Enzyme Linked Immune Sorbent Assay) using a commercial Human Periostin (POSTN) ELISA Kit [6].

\subsection{Blood sampling}

Three ml venous blood was collected from each subject by clean venipuncture using disposable plastic syringe and placed on plain tube (without anticoagulant) for serum separation. The tube was left at room temperature for 30 minutes till coagulation, and then was centrifuged (at 1500 $\mathrm{rpm}$ for 15 minutes).The resultant serum stored at $-20^{\circ} \mathrm{C}$ for further testing.

\subsection{Biochemical analysis}

A double-antibody sandwich ELISA (Enzyme Linked Immune Sorbent Assay) was used to detect serum level of periostin using a commercial Human Periostin (POSTN) ELISA Kit for research use only.The kit was provided by a solid-phase antiPeriostin monoclonal antibody coated to a microtiter plate. When standards and serum samples were added and incubated, the Periostin binds to the solid phase antibodies. The excess antigen washed out and a secondary anti-Periostin antibody labeled with biotin, and combined with Streptavidin-Horse Radish Peroxidase (HRP) enzyme was added and incubated to bind the antibody-antigen complex. After washing completely a TMB (3,3',5,5'- 
Tetramethylbenzidine) substrate solution was added to be catalyzed by the HRP enzyme and give a blue color. The reaction was terminated by adding sulphuric acid $\left(\mathrm{H}_{2} \mathrm{SO}_{4}\right)$ solution where the color changed to yellow and measured spectrophotometrically at a wavelength $450 \mathrm{~nm}$ according to the manufacturer's protocol. The concentration of Periostin was directly proportional to the color intensity of the tested sample. The concentration of Periostin in the samples was then determined by comparing the optical density (O.D.) of the samples to the plotted standard curve.

\subsection{Statistical analysis}

The collected data was revised, coded, tabulated and introduced to a PC using Statistical package for Social Science (IBM Corp. Released 2011. IBM SPSS Statistics for Windows, Version 20.0. Armonk, NY: IBM Corp.). Data were presented and suitable analyses were done according to the type of data obtained for each parameter.

\section{Results and discussion}

Table (1) Comparison serum periostin concentration between all studied groups.

\begin{tabular}{lccc}
\hline & Control & Psoriasis & \multirow{2}{*}{ P } \\
\cline { 2 - 3 } Periostin (ng/ml) & $\mathrm{N}=30$ & $\mathrm{~N}=50$ & \\
\hline Median & 15.1 & 62.1 & $<0.001$ \\
Range & $10.8-39$ & $25-91.3$ & \\
\hline
\end{tabular}

Data are compared using Man Whitny

Psoriasis patients showed higher concentration of serum periostin when compared to control group (p value <0.001) as shown in table 1.

In agreement with our study [7] reported that periostin levels in psoriasis patients is significantly higher than those of normal control and epidermal hyperplasia is a common histological characteristic in psoriasis and periostin is critical for the induction of epidermal hyperplasia [7].

Another study done by [8] found substantial expression of periostin in all investigated psoriasis patients in a pattern similar to $\mathrm{AD}$ patients and that epidermal hyperplasia is decreased in IMQ-treated periostin-deficient mice. These results provide clear evidence that periostin is a mediator for the formation of epidermal hyperplasia common to both $\mathrm{AD}$ and psoriasis.

\section{Conclusion}

The results of our study suggest that periostin may play a role in the pathogenesis of PV and may provide important clues to assist in the development of new therapeutic strategies for psorasis patients.

\section{References}

[1] A. Ahmed, D.C. Butler, A. Leon and J. Reichenberg, Quality-of-life effects of common dermatological diseases. Semi Cuan Med Surg, Vol.32(2),PP.101-109, 2013.

[2] B. Nickoloff and F. Nestle, Recent insights Into the Immunopathogenesis of psoriasis provide new therapeutic opportunities. Journal Clincal Investigation, Vol.113(12),PP.16641675, 2004.
[3] Reich K, The concept of psoriasis as a systemic inflammation: implications for disease management. Journal European Academy Dermatol Venereol, Vol.26, PP. 3-11, 2012.

[4] K. Taniguchi, K.. Arima, M. Masuoka, S. Ohta, H. Shiraishi, K. Ontsuka et al, Periostin controls keratinocyte proliferation and differentiation by interacting with the paracrine IL-1 $\alpha /$ IL-6 loop. Journal Invest Dermatol, Vol.134(5), PP.1295-1304, 2014.

[5] S. Feldman and G. Kruger, psorasis assessment tool in clinical trials.Ann Rheum Dis, Vol.64(2),PP.65-68,2005.

[6] L. Ludemann , W. Grieger, R. Wurm R. P. Wust , C. Zimmer. Glioma assessment using quantitative blood volume maps generated by T1-weighted dynamic contrast-enhanced magnetic resonance imaging: a receiver operating characteristic study. Acta Radiol, Vol.47, PP.303-310,2006.

[7] k. Kou, T. Okawa, Y. Yamaguchi , J. Ono, Y. Inoue, M. Kohno et al, Periostin levels correlate with disease severity and chronicity in patients with atopic dermatitis. British Journal Dermatol, Vol.171( 2), PP.283-91, 2014.

[8] K. Arima, S. Ohta , A.Takagi, H. Shiraishi , M. Masuoka , K.Ontsuka et al, Periostin contributes to epidermal hyperplasia in psoriasis common to atopic dermatitis. Allergol Int, Vol.46(1), PP. 41:8, 2015. 